\title{
Linear spaces, transversal polymatroids and ASL domains
}

\author{
Aldo Conca
}

Received: 7 February 2006 / Accepted: 20 April 2006 /

Published online: 11 July 2006

(C) Springer Science + Business Media, LLC 2007

\begin{abstract}
We study a class of algebras associated with linear spaces and its relations with polymatroids and integral posets, i.e. posets supporting homogeneous ASL. We prove that the base ring of a transversal polymatroid is Koszul and describe a new class of integral posets. As a corollary we obtain that every Veronese subring of a polynomial ring is an ASL.
\end{abstract}

Keywords Families of linear spaces · Transversal polymatroids $\cdot$ Koszul algebras · ASL $\cdot$ Veronese rings · Gröbner bases

\section{Introduction}

Let $K$ be an infinite field and $R=K\left[x_{1}, \ldots, x_{n}\right]$ be a polynomial ring over $K$. Let $V=V_{1}, \ldots, V_{m}$ be a collection of vector spaces of linear forms. Denote by $A(V)$ the $K$-subalgebra of $R$ generated by the elements of the product $V_{1} \ldots V_{m}$. Our goal is to investigate the properties of the algebra $A(V)$ and its relationship with conjectures and questions of White, Herzog and Hibi on polymatroids and with the study of integral posets.

\subsection{Polymatroids}

A finite subset $B$ of $\mathbb{N}^{n}$ is a base set of a discrete polymatroid $P$ if for every $v=$ $\left(v_{1}, \ldots, v_{n}\right), w=\left(w_{1}, \ldots, w_{n}\right) \in B$ one has $v_{1}+\cdots+v_{n}=w_{1}+\cdots+w_{n}$ and for all $i$ such that $v_{i}>w_{i}$ there exists a $j$ with $v_{j}<w_{j}$ and $v+e_{j}-e_{i} \in B$. Here $e_{k}$ denotes the $k$-th vector of the standard basis of $\mathbb{N}^{n}$. The notion of discrete polymatroid is a generalization of the classical notion of matroid, see [9, 11, 18, 25]. Associated

\footnotetext{
A. Conca $(\bowtie)$

Dipartimento di Matematica, Universitá di Genova, Genova, Italy e-mail: conca@dima.unige.it
} 
with the base $B$ of a discrete polymatroid $P$ one has a $K$-algebra $K[B]$, called the base ring of $P$, defined to be the $K$-subalgebra of $R$ generated by the monomials $x^{v}$ with $v \in B$. The algebra $K[B]$ is known to be normal and hence Cohen-Macaulay [11]. White predicted in [26] the shape of the defining equations of $K[B]$ as a quotient of a polynomial ring: they should be the quadrics arising from the so-called symmetric exchange relations of the polymatroids. Herzog and Hibi [11] did not "escape from the temptation" to ask whether $K[B]$ is defined by a Gröbner basis of quadrics and whether $K[B]$ is a Koszul algebra. These two questions are closely related to White's conjecture. This is because for any standard graded algebra $A$ with defining ideal $I$, the existence of a Gröbner basis of quadrics for $I$ implies the Koszul property of $A$ which implies that $I$ is defined by quadrics.

If $C_{1}, \ldots, C_{m}$ are non-empty subsets of $\{1, \ldots, n\}$ then the set of vectors $\sum_{k=1}^{m} e_{j_{k}}$ with $j_{k} \in C_{k}$ is the base of a polymatroid. Polymatroids of this kind are called transversal. Therefore the base rings of transversal polymatroids are exactly the rings of type $A(V)$ where the spaces $V_{i}$ are generated by variables. For transversal polymatroids we prove that the base ring $K[B]$ is Koszul and describe the defining equations, see Section 3. Indeed, $K[B]$ is defined as a quotient of a Segre product $T^{*}$ of polynomial rings by a Gröbner basis of linear binomial forms of $T^{*}$.

\subsection{ASL and integral posets}

Algebras with straightening laws (ASL for short) on posets were introduced by De Concini, Eisenbud and Procesi [7, 10], see also [4]. The abstract definition of an ASL was inspired by earlier work of Hochster, Hodge, Laksov, Musili, Rota, and Seshadri among others. It was motivated by the existence of many families of classical algebras, such as coordinate rings of Grassmannians and their Schubert subvarieties and various kinds of determinantal rings, which could be treated within that framework. We recall in 5.4 the definition of homogeneous ASL and in 5.5 a well-known characterization of them in terms of revlex Gröbner bases.

A finite poset $H$ is integral (with respect to a field $K$ ) if there exists a homogeneous ASL domain supported on $H$. A beautiful result, due to Hibi [14], says that any distributive lattice $L$ is integral. Indeed, $L$ supports a homogeneous ASL domain, denoted by $H_{L}$, in a very natural way. The ring $H_{L}$ is called the Hibi ring of $L$ and its defining equations are the so-called Hibi relations: $x y-(x \wedge y)(x \vee y)$. In a series of papers [15-17, 22, 23] Hibi and Watanabe classified various families of integral posets of low dimension. In this direction, we construct a new class of integral posets: the rank truncations of hypercubes. In details, given a sequence of positive integers $d=d_{1}, \ldots, d_{m}$, let $H(d)=\Pi_{i=1}^{m}\left\{1, \ldots, d_{i}\right\}$ and, for $n \in \mathbb{N}, H_{n}(d)=\{\alpha \in H(d)$ : rk $\alpha<n\}$. We show that $H_{n}(d)$ is an integral poset (over every infinite field $K$ ). This is done by proving that $A(V)$ is a homogeneous ASL on $H_{n}(d)$ if the $V_{i}$ are generic linear spaces of dimension $d_{i}$ of $R$, see Section 5. In particular, our construction shows that the Veronese subrings of polynomials rings are homogeneous ASL (obviously domains). Note however that they are not, in general, ASL with respect to their semigroup presentation.

Results from [6] show that for any collection $V=V_{1}, \ldots, V_{m}$ the algebra $A(V)$ is normal. As said above, in the monomial case, i.e. when $V_{i}$ are generated by variables, we show that $A(V)$ is Koszul and describe its defining equations. Our argument for 
the monomial case is based on a certain elimination process and on a result, Theorem 3.1, proved independently by Sturmfels and Villarreal, describing the universal Gröbner basis of the ideal of 2-minors of a matrix of variables. This approach suggests also a possible strategy for proving that $A(V)$ is Koszul in the general case. The elimination process is still available and what one needs is a replacement of the Sturmfels-Villarreal's theorem. This boils down to the following:

Conjecture 1.1. Let $t_{i j}$ be distinct variables over a field $K$ with $1 \leq i \leq m$ and $1 \leq$ $j \leq n$. Let $L=\left(L_{i j}\right)$ be an $m \times n$ matrix with $L_{i j}=\sum_{k=1}^{n} a_{i j k} t_{i k}$ and $a_{i j k} \in K$ for all $i, j, k$. Denote by $I_{2}(L)$ the ideal of the 2-minors of $L$. We conjecture that for every choice of $a_{i j k}$ 's, and for every term order $<$ on $K\left[t_{i j}\right]$ the initial ideal in ${ }_{<}\left(I_{2}(L)\right)$ is square-free in the $\mathbb{Z}^{m}$-graded sense, i.e. it is generated by elements of the form $t_{i_{1} j_{1}} \ldots t_{i_{k} j_{k}}$ with $i_{1}<i_{2}<\cdots<i_{k}$.

This conjecture can be rephrased in terms of universal comprehensive Gröbner bases [24]: the parametric ideal $I_{2}(L)$ (the parameters being the $a_{i j k}$ 's) has a comprehensive and universal Gröbner basis whose elements are multihomogeneous of degree bounded by $(1,1, \ldots, 1)$.

If $L=\left(t_{i j}\right)$ then 1.1 holds; this is a consequence of Theorem 3.1. We prove in Theorem 5.1 that Conjecture 1.1 holds when $a_{i j k}$ are generic. As a consequence, we are able to show that for generic spaces $V_{i}$ algebra $A(V)$ is Cohen-Macaulay and Koszul, and describe the defining equations of $A(V)$. In particular, as mentioned above, in the generic case $A(V)$ turns out to be a homogeneous ASL on the poset $H_{n}(d)$ where $d=d_{1}, \ldots, d_{m}$ and $d_{i}=\operatorname{dim} V_{i}$.

We thank C. Krattenthaler who provided a combinatorial argument for a statement which was used in an earlier version of the proof of Theorem 5.1. The results presented in this paper have been inspired, suggested and confirmed by computations performed by computer algebra system CoCoA [5].

\section{Normality of $A(V)$}

Let $I_{i}$ be the ideal of $R$ generated by $V_{i}$. In [6] it is proved that the product ideal $I_{1} \ldots I_{m}$ has always a linear resolution. One of the main steps in proving that result is the following $[6,3.2]$ :

Proposition 2.1. For any subset $A \subseteq\{1, \ldots, m\}$ set $I_{A}=\sum_{i \in A} I_{i}$ and denote by \#A the cardinality of $A$. Then

$$
I_{1} \ldots I_{m}=\cap I_{A}^{\# A}
$$

is a primary decomposition of I. Here the intersection is extended to all $A \neq \emptyset$.

Proposition 2.1 easily implies:

Theorem 2.2. $A(V)$ is normal. 
Proof: Set $J=I_{1} \ldots I_{m}$. Note that $I_{A}$ is a prime ideal generated by linear forms. Hence the powers of $I_{A}$ are integrally closed. It follows that $J$ is integrally closed. Since the powers of $J$ are again products of ideals of linear forms, the same argument applies also to the powers of $J$. Hence we conclude that $J$ is normal (i.e. all powers of $J$ are integrally closed). This is equivalent to the fact that the Rees algebra $\mathcal{R}(J)=\oplus_{k \in \mathbb{N}} J^{k}$ is normal. Now $A(V)$, being a direct summand of $\mathcal{R}(J)$, is normal as well.

\section{The monomial case}

We now analyze the monomial case. Our goal is to show that $A(V)$ is Koszul if each $V_{i}$ is monomial and to develop a strategy to attack the general case. So in this section we assume that each $V_{i}$ is generated by a subset of the variables $\left\{x_{1}, \ldots, x_{n}\right\}$. Say $V_{i}=\left\langle x_{j}: j \in C_{i}\right\rangle$ where $C_{i}$ is a non-empty subset of $\{1, \ldots, n\}$. Consider the auxiliary algebra

$$
B(V)=K\left[V_{1} y_{1}, \ldots, V_{m} y_{m}\right]=K\left[y_{i} x_{j}: i \in 1, \ldots, m, \text { and } j \in C_{i}\right]
$$

where $y_{1}, \ldots, y_{m}$ are new variables. The algebra $B(V)$ sits inside the Segre product

$$
S=K\left[y_{i} x_{j}: 1 \leq i \leq m, 1 \leq j \leq n\right]
$$

We consider variables $t_{i j}$ with $i=1, \ldots, m$ and $j=1, \ldots, n$, and define

$$
T=K\left[t_{i j}: 1 \leq i \leq m, 1 \leq j \leq n\right] \quad \text { and } \quad T(V)=K\left[t_{i j}: 1 \leq i \leq m, j \in C_{i}\right]
$$

and the presentations:

$$
\phi: T \rightarrow S \quad \text { and } \quad \phi^{\prime}: T(V) \rightarrow B(V)
$$

are defined by sending $t_{i j}$ to $y_{i} x_{j}$.

It is well-known that $\operatorname{Ker} \phi$ is the ideal $I_{2}(t)$ of 2-minors of the $m \times n$ matrix $t=$ $\left(t_{i j}\right)$. Then the algebra $B(V)$ is defined as a quotient of $T(V)$ by the ideal $I_{2}(t) \cap T(V)$. The algebras $B(V), T(V), S$ and $T$ can be given a $\mathbb{Z}^{m}$-graded structure by setting the degree of $y_{i} x_{j}$ and $t_{i j}$ to be $e_{i} \in \mathbb{Z}^{m}$.

By work of Sturmfels [20, 4.11 and 8.11] and Villarreal [21, 8.1.10 ] one knows that a universal Gröbner basis of $I_{2}(t)$ is given by the cycles of the complete bipartite graph $K_{n, m}$. In details, a cycle of the complete bipartite graph is described by a pair $(I, J)$ of sequences of integers, say

$$
I=i_{1}, \ldots, i_{s}, \quad J=j_{1}, \ldots, j_{s}
$$

with $2 \leq s \leq \min (n, m), 1 \leq i_{k} \leq m, 1 \leq j_{k} \leq n$, and such that the $i_{k}$ are distinct and the $j_{k}$ are distinct. Associated with any such a pair we have polynomial

$$
f_{I, J}=t_{i_{1} j_{1}} \ldots t_{i_{s} j_{s}}-t_{i_{2} j_{1}} \ldots t_{i_{s} j_{s-1}} t_{i_{1} j_{s}}
$$

which is in $I_{2}(t)$. 
Theorem 3.1 (Sturmfels-Villarreal). The set of the polynomials $f_{I, J}$ where $(I, J)$ is a cycle of $K_{n, m}$ forms a universal Gröbner basis of $I_{2}(t)$.

In particular we have:

Corollary 3.2. The polynomials $f_{I, J}$ involving only variables of $T(V)$ form a universal Gröbner basis of $I_{2}(t) \cap T(V)$.

Important for us is the following:

Corollary 3.3. The ideal $I_{2}(t) \cap T(V)$ has a universal Gröbner basis whose elements have $\mathbb{Z}^{m}$-degree bounded above by $(1,1, \ldots, 1) \in \mathbb{Z}^{m}$.

For a $\mathbb{Z}^{m}$-graded algebra $E$ we denote by $E_{\Delta}$ the direct sum of the graded components of $E$ of degree $(v, v, \ldots, v) \in \mathbb{Z}^{m}$ as $v$ varies in $\mathbb{Z}$. Similarly, for a $\mathbb{Z}^{m}$-graded $E$-module $M$ we denote by $M_{\Delta}$ the direct sum of the graded components of $M$ of degree $(v, v, \ldots, v) \in \mathbb{Z}^{m}$ as $v$ varies in $\mathbb{Z}$. Clearly $E_{\Delta}$ is a $\mathbb{Z}$-graded algebra and $M_{\Delta}$ is a $\mathbb{Z}$-graded $E_{\Delta}$-module. Furthermore $-_{\Delta}$ is exact as a functor on the category of $\mathbb{Z}^{m}$-graded $E$-modules with maps of degree 0 .

Now $B(V)_{\Delta}$ is the $K$-algebra generated by the elements in $y_{1} V_{1} \ldots y_{m} V_{m}$. Therefore $A(V)$ is (isomorphic to) the algebra $B(V)_{\Delta}$.

Hence we obtain a presentation

$$
0 \rightarrow Q \rightarrow T^{*} \rightarrow A(V) \rightarrow 0
$$

where $Q=\left(I_{2}(t) \cap T(V)\right)_{\Delta}$ and $T^{*}=T(V)_{\Delta}$ is the $K$-algebra generated by the monomials $t_{1 j_{1}} \ldots t_{m j_{m}}$ with $j_{k} \in C_{k}$, that is, $T^{*}$ is the Segre product of the polynomial rings

$$
T_{i}=K\left[t_{i j}: j \in C_{i}\right]
$$

From Corollary 3.3 we get:

Corollary 3.4. The ideal $Q$ is generated by elements of degree $(1,1, \ldots, 1)$ which form a Gröbner basis with respect to any term order on the variables $t_{i j}$.

Proof: Let $g \in Q$ be a homogeneous element of degree, say, $(a, a, \ldots, a)$. Then there exists $h \in I_{2}(t) \cap T(V)$ of multidegree $\leq(1,1, \ldots, 1)$ such that $\operatorname{in}(h) \mid \operatorname{in}(g)$. Then there exists a monomial $v$ of multidegree $(1,1, \ldots, 1)-\operatorname{deg} h$ such that in $(h) v \mid \operatorname{in}(g)$. It follows that $h v \in Q$ has degree $(1,1, \ldots, 1)$ and its initial term divides in $(g)$.

In 3.4 (and later on) we consider Gröbner bases and initial ideals of ideals in $K$ subalgebras of polynomial rings. For the details on this "relative" Gröbner basis theory the reader can consult, for instance, [2, Section 3] or [20, Chapter 11]. We may now conclude: 
Theorem 3.5. If $V_{i}$ are generated by variables then $A(V)$ is a Koszul algebra. Moreover $A(V)$ is the quotient of the Segre product $T^{*}$ by an ideal generated by linear (binomial) forms which are a Gröbner basis.

Proof: From 3.4 we know that the initial ideal in $(Q)$ (with respect to any term order) is an ideal of $T^{*}$ generated by a subset of the monomials generating $T^{*}$ as a $K$-algebra. By work of Herzog, Hibi and Restuccia $[12,2.3]$ we know that Segre products of polynomial rings are strongly Koszul semigroup rings. Strongly Koszul semigroup rings remain strongly Koszul after moding out by semigroup generators [12, 2.1]. So $T^{*} / \operatorname{in}(Q)$ is strongly Koszul and in particular Koszul. But then the standard deformation argument shows that $T^{*} / Q$ is Koszul, see [2, 3.16] for details. Therefore we can conclude that $A(V)$ is a Koszul algebra.

Remark 3.6. In the proof above we have shown that a Segre product of polynomial rings modulo a certain ideal of linear forms is Koszul. One might ask whether the linear sections of the Segre product of polynomial rings are always Koszul. It is not the case. The ideal of 2-minors of the matrix

$$
\left(\begin{array}{llll}
0 & x & y & z \\
x & y & 0 & t
\end{array}\right)
$$

defines an algebra which is a linear section of the Segre product of polynomial rings of dimension 2 and 4 and it is not Koszul. This is the algebra number 69 in Roos' list [19], a well-known gold-mine of examples.

Keeping track of the various steps of the construction above one can describe the defining equations of $A(V)$. In details, we set $C=C_{1} \times C_{2} \times \cdots \times C_{m}$. Consider variables $s_{\alpha}$ with $\alpha \in C$ and the polynomial ring $K[C]=K\left[s_{\alpha}: \alpha \in C\right]$. Then we get presentations of the Segre product $T^{*}$ and of $A(V)$ as quotients of $K[C]$ by sending $s_{\left(j_{1}, \ldots, j_{m}\right)}$ to $t_{1 j_{1}} \ldots t_{m j_{m}}$ and to $x_{j_{1}} \ldots x_{j_{m}}$ respectively.

The ring $T^{*}$ is the Hibi ring of the distributive lattice $C$ so it is defined by the Hibi relations, namely

$$
s_{\alpha} s_{\beta}-s_{\alpha \vee \beta} s_{\alpha \wedge \beta}
$$

where

$$
\alpha \vee \beta=\left(\max \left(\alpha_{1}, \beta_{1}\right), \ldots, \max \left(\alpha_{m}, \beta_{m}\right)\right)
$$

and

$$
\alpha \wedge \beta=\left(\min \left(\alpha_{1}, \beta_{1}\right), \ldots, \min \left(\alpha_{m}, \beta_{m}\right)\right)
$$

We have: 
Proposition 3.7. The defining ideal of $A(V)$ as the quotient of the polynomial ring $K[C]$ is generated by the Hibi relations $s_{\alpha} s_{\beta}-s_{\alpha \vee \beta} s_{\alpha \wedge \beta}$ and by the relations

$$
s_{\alpha}-s_{\beta}
$$

where $\alpha, \beta \in C$ and one is obtained from the other by the other with a non-trivial permutation.

For instance:

Example 3.8. Let $n=3$ and $V_{1}=\left\langle x_{2}, x_{3}\right\rangle, V_{2}=\left\langle x_{1}, x_{3}\right\rangle, V_{3}=\left\langle x_{1}, x_{2}\right\rangle$. Then $B(V)$ is the quotient of $K\left[t_{12}, t_{13}, t_{21}, t_{23}, t_{31}, t_{32}\right]$ by the polynomial $t_{12} t_{23} t_{31}-t_{13} t_{21} t_{32}$ and then $A(V)$ is the quotient of $K\left[s_{i j k}:(i, j, k) \in\{2,3\} \times\{1,3\} \times\{1,2\}\right]$ by the Hibirelations

$$
\begin{array}{ll}
s_{312} s_{331}-s_{311} s_{332}, & s_{212} s_{311}-s_{211} s_{312}, \\
s_{212} s_{231}-s_{211} s_{232}, & s_{212} s_{331}-s_{211} s_{332}, \\
s_{231} s_{311}-s_{211} s_{331}, & s_{231} s_{312}-s_{211} s_{332}, \\
s_{232} s_{311}-s_{211} s_{332}, & s_{232} s_{312}-s_{212} s_{332}, \\
s_{232} s_{331}-s_{231} s_{332} &
\end{array}
$$

and by the linear relation

$$
s_{231}-s_{312}
$$

Remark 3.9. It is not clear whether the defining ideal of $A(V)$ as the quotient of $K[C]$ has a Gröbner basis of quadrics. The Hibi relations form a Gröbner basis with respect to any revlex linear extension of the partial order on $C$. There are examples where the Hibi relations together with the linear relations defining $A(V)$ are not a Gröbner basis with respect to such revlex linear extensions.

Remark 3.10. In the following special case it turns out that both $B(V)$ and $A(V)$ are defined by Gröbner bases of quadrics as quotients of polynomial rings. For a nested chain of vector spaces of linear forms $V_{1} \supseteq V_{2} \supseteq \cdots \supseteq V_{m}$, we can fix a basis $x_{1}, x_{2}, \ldots, x_{n}$ of $R_{1}$ such that $V_{i}$ is generated by $x_{1}, \ldots, x_{d_{i}}$. Here $d_{1} \geq d_{2} \geq \cdots \geq d_{m}$. It follows that $B(V)$ corresponds to a one-sided ladder determinantal ring, the ladder being the set of points $(i, j)$ with $1 \leq i \leq m$ and $1 \leq j \leq d_{i}$. Furthermore, $A(V)$ coincides with the algebra associated with the principal Borel subset generated by the monomial $\Pi_{i} x_{d_{i}}$. A Gröbner basis of quadrics for $B(V)$ is described in [13] and a Gröbner basis of quadrics for $A(V)$ is described in [8].

In general, however, the algebra $B(V)$ is not defined by quadrics as Example 3.8 shows. White's conjecture [26] predicts the structure of the defining equations of the base ring of a (poly)matroid: they should be quadrics representing the basic symmetric 
exchange relations of the polymatroid. Our result above Proposition 3.7 does not prove White's conjecture in this precise form.

\section{Conjectures}

The constructions and arguments of the previous section suggest a general strategy to investigate the Koszul property of $A(V)$ for general (i.e. non-monomial) $V_{i}$. We outline in this section the strategy which leads us to Conjecture 1.1. Let $V=V_{1}, \ldots, V_{m}$ be a collection of subspaces of $R_{1}$ and let $y_{1}, \ldots, y_{m}$ be new variables. Set $d_{i}=\operatorname{dim} V_{i}$, and set

$$
\begin{aligned}
S & =K\left[y_{i} x_{j}: i=1, \ldots, m, j=1, \ldots, n\right] \\
B(V) & =K\left[y_{1} V_{1}, \ldots, y_{m} V_{m}\right] .
\end{aligned}
$$

and

$$
T=K\left[t_{i j}: i=1, \ldots, m, j=1, \ldots, n\right]
$$

Again $B(V)$ is a $K$-subalgebra of $S$. We give degree $e_{i} \in \mathbf{Z}^{m}$ to $y_{i} x_{j}$ and to $t_{i j}$ so that $S, T$ and $B(V)$ are $\mathbf{Z}^{m}$-graded. We present $S$ as a quotient of $T$ by sending $t_{i j}$ to $y_{i} x_{j}$. The kernel of such presentation is the ideal $I_{2}(t)$ generated by the 2-minors of the $m \times n$ matrix $t=\left(t_{i j}\right)$. As we have seen in the previous section $A(V)$ is the diagonal algebra $B(V)_{\Delta}$.

We want to get the presentations of $B(V)$ and $A(V)$ by elimination from that $S$. To that end we do the following: Let $f_{i j}, j=1, \ldots, d_{i}$, be a basis of $V_{i}$ and complete it to a basis of $R_{1}$ with elements $f_{i j}, j=d_{i}+1, \ldots, n$. Denote by $f_{i}$ the row vector $\left(f_{i j}\right)$ and by $x$ the row vector of the $x_{i}$ 's. Let $A_{i}$ be the $n \times n$ matrix with entries in $K$ with $x=f_{i} A_{i}$. Then $S=K\left[y_{i} f_{i j}: i=1, \ldots, m, j=1, \ldots, n\right]$ and $B(V)=K\left[y_{i} f_{i j}\right.$ : $\left.i=1, \ldots, m, j=1, \ldots, d_{i}\right]$. Set $T(V)=K\left[t_{i j}: 1 \leq i \leq m, 1 \leq j \leq d_{i}\right]$. We have presentations:

$$
\begin{array}{lll}
\phi: T \rightarrow S & \text { with } t_{i j} \rightarrow y_{i} f_{i j} & \text { for all } i, j \\
\phi^{\prime}: T(V) \rightarrow B(V) & \text { with } t_{i j} \rightarrow y_{i} f_{i j} & \text { for all } i \text { and } 1 \leq j \leq d_{i}
\end{array}
$$

By construction, the kernel of $\phi$ is the ideal of 2-minors $I_{2}(L)$ of the matrix $L=$ $\left(L_{i j}\right)$ where the row vector $\left(L_{i j}: j=1, \ldots, n\right)$ is given by $\left(t_{i 1}, \ldots, t_{i n}\right) A_{i}$. Clearly, $\operatorname{Ker} \phi^{\prime}=I_{2}(L) \cap T(V)$. As explained in the previous section, by applying the diagonal functor we obtain a presentation:

$$
A(V) \simeq T^{*} / Q
$$

where $T^{*}$ is the Segre product of the $T_{i}$ 's, $T_{i}=K\left[t_{i j}: j=1, \ldots, d_{i}\right]$, and $Q=$ $\left(I_{2}(L) \cap T(V)\right)_{\Delta}$. 
Remark 4.1. One can easily check that the arguments of Section 3, in particular those of 3.4 and 3.5, work and can be used to show that $A(V)$ is Koszul provided one knows that $I_{2}(L) \cap T(V)$ has an initial ideal generated in degree $\leq(1,1, \ldots, 1) \in \mathbb{Z}^{m}$. On the other hand, $I_{2}(L) \cap T(V)$ has the desired initial ideal provided $I_{2}(L)$ has an initial ideal generated in degree $\leq(1,1, \ldots, 1) \in \mathbb{Z}^{m}$ with respect to the appropriate elimination order.

We are led by Remark 4.1 to analyze the initial ideals of ideals of 2-minors of matrices such as $L$. To our great surprise, the experiments support Conjecture 1.1. What we really need is a weak form of Conjecture 1.1, namely:

Conjecture 4.2. Let $L=\left(L_{i j}\right)$ be an $n \times m$ matrix with $L_{i j}=\sum_{k=1}^{n} a_{i j k} t_{i k}$ and $a_{i j k} \in K$ for all $i, j, k$. Assume that for every $i$ the forms $L_{i 1}, \ldots, L_{i n}$ are linearly independent. Then any lexicographic initial ideal of $I_{2}(L)$ is generated in degree $\leq(1,1, \ldots, 1)$.

If conjecture 4.2 holds then from the discussion above it follows that for every $V_{1}, \ldots, V_{m}$ the algebra $A(V)$ is Koszul and defined by a Gröbner basis of linear forms as the quotient of the Segre product $T^{*}$.

The next section is devoted to proving Conjecture 1.1 in the generic case.

\section{The generic case}

We consider now the case of generic spaces $V_{1}, \ldots, V_{m}$. What we prove is the following:

Theorem 5.1. If the matrix $L$ is generic, that is, every entry $L_{i j}=\sum_{k=1}^{n} a_{i j k} t_{i k}$ is a generic linear combination of $t_{i 1}, \ldots, t_{i n}$, then Conjecture 1.1 holds.

The key lemma is:

Lemma 5.2. Let $V_{1}, \ldots, V_{m}$ be subspaces of $R_{1}$. If $\sum_{i=1}^{m} \operatorname{dim} V_{i} \geq n+m$ then $\operatorname{dim} \prod_{i=1}^{m} V_{i}<\prod_{i=1}^{m} \operatorname{dim} V_{i}$, i.e. there is a non-trivial linear relation among the generators of the product $\prod_{i=1}^{m} V_{i}$ obtained by multiplying $K$-bases of the $V_{i}$.

Proof: By induction on $n$ and $m$. If one of the $V_{i}$ is principal then we can simply skip it. The case $m=2$ is easy: the assumption is equivalent to $\operatorname{dim}\left(V_{1} \cap V_{2}\right) \geq 2$ and for $f, g \in V_{1} \cap V_{2}$ we get the non-trivial relation $f g-g f=0$. For $m>2$, if $\operatorname{dim}\left(V_{i} \cap V_{j}\right) \geq 2$ for some $i \neq j$ then the non-trivial relation above gives a nontrivial relation also for $V_{1} \ldots V_{m}$. Therefore we may assume that $\operatorname{dim}\left(V_{i} \cap V_{j}\right)<2$, and, since none of the $V_{i}$ is principal, also none of the $V_{i}$ is $R_{1}$. The case $n=2$ follows and to prove the assertion in the general case we may assume that $1<d_{i}<n$ for all $i$. Further we may assume also that the $V_{i}$ are generic, since the dimension of $V_{1} \ldots V_{m}$ for special $V_{i}$ can be only smaller. By genericity of the $V_{i}$ we may find $K$-bases $f_{i j}$ of $V_{i}$ so that any set of $n$ elements in the set $\left\{f_{i j}: i=1, \ldots, m\right.$, and $\left.j=1, \ldots, d_{i}\right\}$ is a basis of $R_{1}$. Now let $x$ be a general linear form (it suffices that $x$ is not contained 
in any sum of the $V_{i}$ which is a proper subspace of $R_{1}$ ). Since $x \notin V_{i}$ we have that $\operatorname{dim} V_{i}+(x) /(x)=d_{i}$, so by induction on $n$ we may find a non-trivial relation among the generators of $V_{1} \ldots V_{m}$ modulo $x$. In other words there exists a relation of the form

$$
\sum \lambda_{\alpha} f_{1 \alpha_{1}} \ldots f_{m \alpha_{m}}=x h
$$

where $\lambda_{\alpha} \in K$, the sum is extended over all $\alpha$ in $\prod_{i=1}^{m}\left\{1, \ldots, d_{i}\right\}$ and at least one of the $\lambda_{\alpha}$ is non-zero. We may assume $\lambda_{\alpha} \neq 0$ for $\alpha=(1,1, \ldots, 1)$. By the above relation we have that $x h \in \prod_{i=1}^{m} V_{i}$ and hence $x h \in \prod_{i \neq j} V_{i}$ for all $j$. But from Proposition 2.1 we see immediately that $x$ acts as a non-zero divisor in degree $m-1$ and higher on the ideal generated by $\prod_{i \neq j} V_{i}$. It follows that $h \in \prod_{i \neq j} V_{i}$ for all $j$. By the choice of the $f_{i j}$ and since $\sum_{i=1}^{m} d_{i} \geq n+m$ we may write $x$ as a linear combination of the $f_{i j}$ with $i=1, \ldots, m$, and $1<j \leq d_{i}$. It follows that $x h$ can be written as a linear combination of the $f_{1 \alpha_{1}} \ldots f_{m \alpha_{m}}$ with $\alpha \neq(1,1, \ldots, 1)$. Hence we obtain a relation

$$
\sum \lambda_{\alpha}^{\prime} f_{1 \alpha_{1}} \ldots f_{m \alpha_{m}}=0
$$

with $\lambda_{\alpha}^{\prime}=\lambda_{\alpha} \neq 0$ for $\alpha=(1,1, \ldots, 1)$.

Now we are ready to prove:

Proof of Theorem 5.1: Set $I=I_{2}(L)$. Let $<$ be a term order on the $t_{i j}$. After a name change of the variables in the $i$-th row of $L$ if needed, we may assume that $t_{i j+1}>t_{i j}$ for all $j=1, \ldots, n-1$ and for all $i=1, \ldots, m$. Let $J$ be the ideal generated by the monomials

$$
t_{i_{1} j_{1}} \ldots t_{i_{k} j_{k}}
$$

satisfying conditions:

$$
(*)\left\{\begin{array}{l}
1 \leq i_{1}<\cdots<i_{k} \leq m \\
1 \leq j_{1}, \ldots, j_{k} \leq n \\
j_{1}+\cdots+j_{k} \geq n+k
\end{array}\right.
$$

We will show that the initial ideal of $I$ with respect to $<$ is equal to $J$. From this the assertion follows immediately. It is a simple exercise on primary decompositions that the equality $J=\operatorname{in}(I)$ follows from three facts:

(1) $J \subseteq \operatorname{in}(I)$,

(2) $J$ and $I$ have the same codimension and degree,

(3) $J$ is unmixed.

For (1) we have to show that for each pair of sequences of integers satisfying conditions $(*)$ the monomial $t_{i_{1} j_{1}} \ldots t_{i_{k} j_{k}}$ is in in $(I)$. As $L$ is generic, the initial ideal $\operatorname{in}(I)$ is the multigraded generic initial ideal of $I$ with respect to $>$. Hence in $(I)$ is Borel fixed in the multigraded sense, see [1]. In characteristic 0 this means that if a monomial $M$ is in in $(I)$ and $t_{i j} \mid M$ then $t_{i k} M / t_{i j}$ is in in(I) as well for all the $k>j$. 
In arbitrary characteristic the same assertion is also true as long as $M$ is square-free. It follows that (no matter what the characteristic is) it suffices to show that there exists an $f$ in $I$ such that $\operatorname{in}(f)=t_{i_{1} p_{1}} \ldots t_{i_{k} p_{k}}$ and $p_{1} \leq j_{1}, \ldots, p_{k} \leq j_{k}$. To this end, consider the linear forms $f_{i j}$ defined (implicitly) by the relation $x_{j}=\sum_{k=1}^{n} f_{i j} a_{i k j}$ for all $j$. By the construction of Section 4 we see that $I$ is the kernel of the map $\phi$. Now for $s=1, \ldots, k$ consider the subspace $W_{i_{s}}$ generated by the $f_{i_{s} j}$ with $j \leq j_{s}$. Since, by assumption $\sum_{s=1}^{k} \operatorname{dim} W_{i_{s}}=\sum_{s=1}^{k} j_{s} \geq n+k$, by Lemma 5.2 we have that there exists a non-trivial relation among the generators of the product $W_{i_{1}} \ldots W_{i_{k}}$. This implies that $I$ contains a non-zero polynomial $f$ supported on the set of monomials $t_{i_{1} p_{1}} \ldots t_{i_{k} p_{k}}$ where $p_{1} \leq j_{1}, \ldots, p_{k} \leq j_{k}$. Take in $(f)$ to get what we want.

As for the steps (2) and (3), the ideal $I$ is a generic determinantal ideal and its numerical invariants are well-known: its codimension is $(m-1)(n-1)$ and its degree is $\left(\begin{array}{c}m+n-2 \\ m-1\end{array}\right)$. Knowing the generators of $J$ we can describe the facets of the associated simplicial complex $\Delta(J)$. Then we can read from the descriptions of the facets the codimension of $J$ and check that it is unmixed. The facets of $\Delta(J)$ have the following description: for each $p=\left(p_{1}, \ldots, p_{m}\right) \in\{1, \ldots, n\}^{m}$ with $p_{1}+\cdots+p_{m}=n+m-1$ we let

$$
F_{p}=\left\{t_{i j}: i=1, \ldots, m \text { and } 1 \leq j \leq p_{i}\right\}
$$

It is easy to check that any such $F_{p}$ is a facet of $\Delta(J)$. On the other hand if $F$ is a face of $\Delta(J)$ let $a(F)=\left\{i: \exists j\right.$ with $\left.t_{i j} \in F\right\}$ and $j_{i}=\max \left\{j: t_{i j} \in F\right\}$ if $i \in a(F)$. Then set $q=\left(q_{1}, \ldots, q_{m}\right)$ with $q_{i}=j_{i}$ if $a \in a(F)$ and $q_{i}=1$ otherwise. Note that

$$
q_{1}+\cdots+q_{m}=\sum_{i \in a(F)} j_{i}+m-|a(F)|
$$

and that

$$
\sum_{i \in a(F)} j_{i}<n+|a(F)|
$$

since $\left\{t_{i j_{i}}: i \in a(F)\right\} \subset F \in \Delta(J)$. It follows that $q_{1}+\cdots+q_{m}<n+m$. So, increasing the $q_{i}$ 's if needed, we may take $p=\left(p_{1}, \ldots, p_{m}\right) \in\{1, \ldots, n\}^{m}$ with $p_{1}+\cdots, p_{m}=n+m-1$ and $q_{i} \leq p_{i}$. It follows that $F \subseteq F_{p}$.

From the description above we see that the cardinality of each $F_{p}$ is $n+m-1$. It follows that $J$ is unmixed of codimension $(m-1)(n-1)$. The degree of $J$ is the number of facets of $\Delta(J)$, that is the number of $p=\left(p_{1}, \ldots, p_{m}\right) \in\{1, \ldots, n\}^{m}$ with $p_{1}+\cdots+p_{m}=n+m-1$. Setting $q_{i}=p_{i}-1$, we see that the number of facets of $\Delta(J)$ is the number of $q=\left(q_{1}, \ldots, q_{m}\right) \in\{0, \ldots, n-1\}^{m}$ with $q_{1}+\cdots+q_{m}=n-1$, that is, the number of monomials of degree $n-1$ in $m$ variables. This number is $\left(\begin{array}{c}m+n-2 \\ m-1\end{array}\right)$. We have checked that (2) and (3) hold. The proof of the theorem is now complete.

Let us single out the following corollary of the proof of Theorem 5.1:

Corollary 5.3. With the notation of the proof of Theorem 5.1 we have: 
(a) If $i_{1}<\cdots<i_{k}$ then a monomial $t_{i_{1} j_{1}} \ldots t_{i_{k} j_{k}}$ is in $J$ iff $j_{1}+\cdots+j_{k} \geq n+k$.

(b) For every monomial $M=t_{i_{1} j_{1}} \ldots t_{i_{k} j_{k}} \in J$ with $i_{1}<\cdots<i_{k}$ there exists a polynomial $f_{M} \in I$ of the form

$$
f_{M}=M+\sum_{v} \lambda_{v} t_{i_{1} v_{1}} \ldots t_{i_{k} v_{k}}
$$

where $\lambda_{v} \in K, v \in \Pi_{h=1}^{k}\left\{1,2, \ldots, j_{h}\right\}$, and $t_{i_{1} v_{1}} \ldots t_{i_{k} v_{k}} \notin J$.

(c) The set of the polynomials $f_{M}$ is a Gröbner basis of I with respect to any term order $<$ on $K\left[t_{i j}\right]$ satisfying $t_{i j+1}>t_{i j}$ for all $j=1, \ldots, n-1$ and all $i=1, \ldots, m$.

Proof: (a) follows from the definition of $J$. For (b) we argue as follows. Let $<$ be a term order on $K\left[t_{i j}\right]$ satisfying $t_{i j+1}>t_{i j}$ for all $j=1, \ldots, n-1$ and for all $i=1, \ldots, m$. We have seen in the proof of Theorem 5.1 that $J=\mathrm{in}_{<}(I)$. Considering the reduced expression, we have that for every monomial $M=t_{i_{1} j_{1}} \ldots t_{i_{k} j_{k}} \in J$ there exists a polynomial $f_{M}$ in $I$ with initial term $M$ and all the others terms not in $J$. Suppose that one of the non-leading terms of $f_{M}$, say $N=t_{i_{1} v_{1}} \ldots t_{i_{k} v_{k}}$, does not satisfy the condition $v_{h} \leq j_{h}$ for some $h=1, \ldots, k$. So there exists an $h$ in $\{1,2, \ldots, k\}$, say $h_{1}$, such that $v_{h_{1}}>j_{h_{1}}$. We claim that there exists a term order $<_{1}$ such that $t_{i j+1}>_{1} t_{i j}$ for all $i, j$ and such that $N>_{1} M$. Then it follows that the initial term of $f_{M}$ with respect to $<_{1}$ is not $M$ and hence it must be a monomial not in $J$. This contradicts the fact, proved in 5.1 that in $<_{1}(I)=J$. It remains to prove the existence of a term order $<_{1}$ as above. To this end it suffices to find weights $w_{i j} \in \mathbb{N}$ such that $w_{i j}<w_{i j+1}$ for all $i, j$ and $w(M)<w(N)$, that is

$$
w_{i_{1}, j_{1}}+\cdots+w_{i_{k} j_{k}}<w_{i_{1} v_{1}}+\cdots+w_{i_{k} v_{k}} .
$$

Just take $w_{i j}=j$ if $i \neq i_{h_{1}}$ or $i=i_{h_{1}}$ and $j<v_{h_{1}}$; otherwise take $w_{i j}=a+j$ with $a$ large enough. Finally (c) is a direct consequence of (b).

As explained in Section 4 it follows from Theorem 5.1 that $A(V)$ is Koszul for generic $V$. To get more precise information about the structure of $A(V)$ we analyze in detail the defining equations of $B(V)$ and $A(V)$. To this end we recall the definition of homogeneous ASL on posets.

Let $(H,>)$ be a finite poset and denote by $K[H]$ the polynomial ring whose variables are the elements of $H$. Let $J_{H}$ be the monomial ideal of $K[H]$ generated by $x y$ with $x, y \in H$ such that $x$ and $y$ are incomparable in $H$.

Definition 5.4. Let $A=K[H] / I$ where $I$ is a homogeneous ideal (with respect to the usual grading). One says that $A$ is a homogeneous ASL on $H$ if

(ASL1) The (residue classes of the) monomials not in $J_{H}$ are linearly independent in A.

(ASL2) For every $x, y \in H$ such that $x$ and $y$ are incomparable the ideal $I$ contains a polynomial of the form

$$
x y-\sum \lambda z t
$$

with $\lambda \in K, z, t \in H, z \leq t, z<x$ and $z<y$. 
A linear extension of the poset $(H,<)$ is a total order $<_{1}$ on $H$ such that $x<_{1} y$ if $x<y$. A revlex term order $\tau$ on $K[H]$ is said to be a revlex linear extension of $<$ if $\tau$ induces on $H$ a linear extension of $<$. For obvious reasons, if $A=K[H] / I$ is a homogeneous ASL on $H$ and $\tau$ is a revlex linear extension of $<$ then the polynomials in (ASL2) form a Gröbner basis of $I$ and $\operatorname{in}_{\tau}(I)=J_{H}$. In a sense the converse is also true:

Lemma 5.5. Let $A=K[H] / I$ where I is a homogeneous ideal. Assume that for every revlex linear extension $\tau$ of $<$ one has $\operatorname{in}_{\tau}(I)=J_{H}$. Then $A$ is an ASL on $H$.

Proof: Let $\tau$ be a revlex linear extension of $<$. Since in $\tau(I)=J_{H}$ the monomials not in $J_{H}$ form a $K$-basis of $A$, hence (ASL1) is satisfied. Let $x, y \in H$ be incomparable elements. Then $x y \in \operatorname{in}_{\tau}(I)$ and hence there exists $F \in I$ with $\operatorname{in}_{\tau}(F)=x y$. We can take $F$ reduced in the sense that $x y$ is the only term in $F$ belonging to $J_{H}$. It follows that $F$ has the form

$$
x y-\sum \lambda z t
$$

with $\lambda \in K, z, t \in H$ and $z \leq t$. Assume, by contradiction that this polynomial does not satisfy the conditions required in (ASL2). Then there exist a non-leading term $z_{1} t_{1}$ appearing in $F$ such that $z_{1} \nless x$ or $z_{1} \nless y$. Say $z_{1} \nless x$. It is easy to see that one can find a linear extension $<_{1}$ of $<$ such that $x<_{1} z_{1}$. Denote by $\sigma$ the revlex term order associated with $<_{1}$. Then $x y$ is smaller than $z_{1} t_{1}$ with respect to $\sigma$ and hence $\operatorname{in}_{\sigma}(F)$ is a term not in $J_{H}$, contradicting the assumption.

For a given sequence of positive integers $d=d_{1}, \ldots, d_{m}$ we set

$$
H(d)=\left\{1, \ldots, d_{1}\right\} \times \cdots \times\left\{1, \ldots, d_{m}\right\}
$$

and note that $H(d)$ is a sublattice of $\mathbb{N}^{m}$ with respect to the natural partial order $\alpha \leq \beta$ iff $\alpha_{i} \leq \beta_{i}$ for all $i$. The rank rk $\alpha$ of an element $\alpha=\left(\alpha_{i}\right) \in H(d)$ is $\alpha_{1}+\cdots+\alpha_{m}-m$.

Set

$$
H_{n}(d)=\{\alpha \in H(d): \operatorname{rk} \alpha<n\}
$$

With the notation of Section 4 we have a presentation $\phi^{\prime}: T(V) \rightarrow B(V)$ where $T(V)=K\left[t_{i j}: i=1, \ldots, m, \quad j=1, \ldots, d_{i}\right]$. As a corollary of Theorem 5.1 , by elimination we obtain the following description of $\operatorname{Ker} \phi^{\prime}$ :

Corollary 5.6. Let $V_{1}, \ldots, V_{m}$ be generic spaces of dimension $d_{1}, \ldots, d_{m}$ and let $f_{i j}$ with $j=1, \ldots, d_{i}$ be generic generators of $V_{i}$. Let $<$ be a term order such that $t_{i j}<$ $t_{i j+1}$. Then the ideal Ker $\phi^{\prime}$ has a Gröbner basis whose elements are the polynomials $f_{M}$ of Corollary 5.3 where $M=t_{i_{1} j_{1}} \ldots t_{i_{k} j_{k}}$ with $i_{1}<\cdots<i_{k}, 1 \leq j_{h} \leq d_{i_{h}}$ and $j_{1}+\cdots+j_{k} \geq n+k$. 
Set $T_{i}=K\left[t_{i j}: 1 \leq j \leq d_{i}\right]$ and denote by $T^{*}$ the Segre product $T_{1} * \cdots * T_{m}$. Consider variables $s_{\alpha}$ with $\alpha \in H(d)$ and the polynomial ring $K\left[s_{\alpha}: \alpha \in H(d)\right]$. For each $\alpha \in H(d)$ set $t_{\alpha}=t_{1 \alpha_{1}} \ldots t_{m \alpha_{m}}$.

We get a presentation $K\left[s_{\alpha}: \alpha \in H(d)\right] \rightarrow T^{*}$ by sending $s_{\alpha}$ to $t_{\alpha}$ whose kernel is generated by the Hibi relations:

$$
s_{\alpha} s_{\beta}-s_{\alpha \vee \beta} s_{\alpha \wedge \beta} .
$$

Adopting the notation of Section 4 we get a presentation $A(V)=T^{*} / Q$. To describe the generators of $Q$ we do the following. For every $\alpha \in H(d) \backslash H_{n}(d)$ consider the polynomial $f_{M}$ of Corollary 5.3 associated with the monomial $M=t_{\alpha}$. Set $L_{\alpha}=f_{M}$. So for all $\alpha \in H(d) \backslash H_{n}(d)$ we have

$$
L_{\alpha}=t_{\alpha}-\sum_{\beta<\alpha} \lambda_{\alpha \beta} t_{\beta} \quad \text { with } \lambda_{\alpha \beta} \in K
$$

and the arguments of Corollary 3.4 show that the $L_{\alpha}$ 's form a Gröbner basis of $Q$ for any term order such that $t_{i j}>t_{i j-1}$ for all $i, j$. It follows that

$$
\operatorname{in}(Q)=\left(t_{\alpha}: \alpha \in H(d) \backslash H_{n}(d)\right)
$$

for any term order such that $t_{i j}>t_{i j-1}$ for all $i, j$. Then $T^{*} / \operatorname{in}(Q)$ is defined as the quotient of $K\left[s_{\alpha}: \alpha \in H(d)\right]$ by:

(1) the Hibi relations $s_{\alpha} s_{\beta}-s_{\alpha \vee \beta} s_{\alpha \wedge \beta}$ with $\alpha, \beta \in H(d)$ incomparable.

(2) $s_{\alpha}$ with $\alpha \in H(d) \backslash H_{n}(d)$.

It is easy to see that the elements of type (1) and (2) form a Gröbner basis for any revlex linear extension of the partial order on $H(d)$. Hence a $K$-basis of $T^{*} / \operatorname{in}(Q)$ is given by the monomials not in $J_{H_{n}(d)}+\left(H(d) \backslash H_{n}(d)\right)$. This in turn implies that the Hibi relations and the relation $L_{\alpha}$ form a Gröbner basis of the defining ideal of $A(V)$ (as a quotient of $K\left[s_{\alpha}: \alpha \in H(d)\right]$ by the map sending $s_{\alpha}$ to $f_{1 \alpha_{1}} \ldots f_{m \alpha_{m}}$ ) with respect to any revlex linear extension of the partial order on $H(d)$. Summing up, we have:

Theorem 5.7. Let $V_{1}, \ldots, V_{m}$ be generic spaces of dimension $d_{1}, \ldots, d_{m}$ and take generic generators $f_{i j}$ of $V_{i}$. Then:

(1) We have a surjective $K$-algebra homomorphism $F: K\left[s_{\alpha}: \alpha \in H_{n}(d)\right] \rightarrow A(V)$ sending the variable $s_{\alpha}$ to $f_{1 \alpha_{1}} \ldots f_{m \alpha_{m}}$.

(2) $\operatorname{Ker} F$ is generated by two types of polynomials:

(a)

$$
s_{\alpha} s_{\beta}-s_{\alpha \vee \beta} s_{\alpha \wedge \beta}
$$

if $\alpha, \beta \in H_{n}(d)$ are incomparable and $\alpha \vee \beta \in H_{n}(d)$.

(b)

$$
s_{\alpha} s_{\beta}-\sum \lambda_{\gamma} s_{\gamma} s_{\alpha \wedge \beta}
$$


if $\alpha, \beta \in H_{n}(d)$ are incomparable $\alpha \vee \beta \notin H_{n}(d)$ the sum is extended to the $\gamma \in H_{n}(d)$ with $\gamma \leq \alpha \vee \beta$ and $\lambda_{\gamma} \in K$ (and depends also on $\alpha$ and $\beta$ ).

(3) The polynomials of type (a) and (b) form a Gröbner basis of Ker $F$ with respect to any revlex linear extension of the partial order of $H_{n}(d)$.

(4) $A(V)$ is a homogeneous $A S L$ on the poset $H_{n}(d)$.

(5) $A(V)$ is normal, Cohen-Macaulay and Koszul.

(6) $A(V)$ is defined, as the quotient of the Segre product $T^{*}$, by a Gröbner basis of linear forms.

(7) The Krull dimension of $A(V)$ is $\min \left\{n, \operatorname{dim} T^{*}=1-m+\sum_{i=1}^{m} d_{i}\right\}$ and its degree is the number of maximal chains in $H_{n}(d)$.

Proof: (1), (2), (3) and (6) follow immediately from the discussion above and (4) follows from Lemma 5.5 and (3). As for (5), normality is proved in Theorem 2.2, Koszulness follows from the general argument of Section 4 and also from (3). The Cohen-Macaulay property and (7) follow from (4) by applying [4, Chap. 5] since $H_{n}(d)$ is a wonderful poset.

As a corollary we obtain:

Corollary 5.8. For every $m$ and $n$, the Veronese subring $R^{(m)}$ of $R=K\left[x_{1}, \ldots, x_{n}\right]$ is an ASL on the poset $H_{n}(d)$ where $d=n, n, \ldots, n$ (m-times).

Remark 5.9. The realization of the $m$-th Veronese subring of a polynomial ring in $n$ variables as a homogeneous ASL has been done before for $n=2$ and any $m$ in [22], for $n=m=3$ in [15] and in two different ways, and for $n=m=4$ in [23].

An interesting consequence of Corollary 5.6 is:

Corollary 5.10. Let $V_{1}, \ldots, V_{m}$ be subspaces of $R_{1}$ of dimension $d_{1}, d_{2}, \ldots, d_{m}$ then:

(a) $\operatorname{dim} \Pi_{i=1}^{m} V_{i} \leq\left|H_{n}(d)\right|$.

(b) if the $V_{i}$ are generic then $\operatorname{dim} \Pi_{i=1}^{m} V_{i}=\left|H_{n}(d)\right|$.

(c) if the $V_{i}$ are generic and if $f_{i j}$ with $j=1, \ldots, d_{i}$ are generic generators of $V_{i}$ then the set $\left\{f_{1 j_{1}} \ldots f_{m j_{m}}:\left(j_{1}, \ldots, j_{m}\right) \in H_{n}(d)\right\}$ is a $K$-basis of $\Pi_{i=1}^{m} V_{i}$.

(d) if the $V_{i}$ are generic then: $\operatorname{dim} \prod_{i=1}^{m} V_{i}=\prod_{i=1}^{m} \operatorname{dim} V_{i}$ iff $\sum \operatorname{dim} V_{i}<m+n$.

Proof: Obviously (b) implies (a) and also (c) implies (b) and (d). So we only have to prove (c). By definition, the product $\Pi_{i=1}^{m} V_{i}$ is the component of degree $(1,1, \ldots, 1)$ of the algebra $B(V)$. Then the conclusion follows from 5.6.

Example 5.11. Take $n=3, d_{1}=d_{2}=d_{3}=2$ and generic spaces $V_{i}$ of dimension $d_{i}$. Note that, up to a choice of coordinates, we are in the situation of Example 3.8 and so the structure of $A(V)$ has been already identified. But to describe the ASL structure of $A(V)$ we have to take generic coordinates for $V_{i}$, say $V_{i}=\left\langle f_{i 1}, f_{i, 2}\right\rangle$. In this case 
$H_{n}(d)$ is the cube $\{1,2\}^{3}$ without the point $(2,2,2)$. We have a relation

$$
f_{12} f_{22} f_{32}=\sum_{\alpha \in H_{n}(d)} \lambda_{\alpha} f_{1 \alpha_{1}} f_{2 \alpha_{2}} f_{3 \alpha_{3}} .
$$

Set $L=\sum_{\alpha \in H_{n}(d)} \lambda_{\alpha} s_{\alpha}$. Then the defining equations of $A(V)$ as the quotient of $K\left[s_{\alpha}\right.$ : $\left.\alpha \in H_{n}(d)\right]$ are:

$$
\begin{array}{lll}
s_{112} s_{221}-s_{111} L, & s_{121} s_{212}-s_{111} L, & s_{211} s_{122}-s_{111} L, \\
s_{121} s_{211}-s_{111} s_{221}, & s_{112} s_{211}-s_{111} s_{212}, & s_{112} s_{121}-s_{111} s_{122}, \\
s_{212} s_{221}-s_{211} L, & s_{122} s_{221}-s_{121} L, & s_{122} s_{212}-s_{112} L
\end{array}
$$

Remark 5.12. With an argument similar to that of 2.2 one can prove that the algebra $B(V)$ is normal for any $V=V_{1}, \ldots, V_{m}$. Furthermore, in the monomial and in the generic case one can prove that $B(V)$ is Cohen-Macaulay. In the monomial case the Cohen-Macaulayness is a consequence of the normality. In generic case it follows from the fact that, by 5.1, we can describe an initial ideal of its defining ideal and such an initial ideal turns out to be associated with a shellable simplicial complex.

\section{References}

1. A. Aramova, K. Crona, and E. De Negri, "Bigeneric initial ideals, diagonal subalgebras and bigraded Hilbert functions," J. Pure Appl. Algebra 150(3) (2000), 215-235.

2. W. Bruns and A. Conca, "Gröbner bases and determinantal ideals," Commutative Algebra, Singularities and Computer Algebra (Sinaia, 2002), pp. 9-66, NATO Sci. Ser. II Math. Phys. Chem., 115, Kluwer Acad. Publ., Dordrecht, 2003.

3. W. Bruns and J. Herzog, Cohen-Macaulay Rings, Cambridge University Press, Cambridge, 1996.

4. W. Bruns and U. Vetter, Determinantal rings, Lecture Notes in Mathematics, 1327. Springer-Verlag, Berlin, 1988.

5. CoCoA Team, CoCoA: A System for doing Computations in Commutative Algebra, Available at http://cocoa.dima.unige.it.

6. A. Conca and J. Herzog "Castelnuovo-Mumford regularity of products of ideals," Collect. Math. 54(2) (2003), 137-152.

7. C. De Concini, D. Eisenbud, and C. Procesi, "Hodge algebras," Astérisque, 91. Société Mathématique de France, Paris, 1982, p. 87.

8. E. De Negri, "Toric rings generated by special stable sets of monomials," Math. Nachr. 203 (1999), $31-45$.

9. J. Edmonds, "Submodular functions, matroids, and certain polyhedra," in Combinatorial Structures and Their Applications, R. Guy, H. Hanani, N. Sauer, and J. Schonheim (Eds.), Gordon and Breach, New York, 1970, pp. 69-87.

10. D. Eisenbud, "Introduction to algebras with straightening laws," Ring Theory and Algebra, III (Proc. Third Conf., Univ. Oklahoma, Norman, Okla., 1979), pp. 243-268, Lecture Notes in Pure and Appl. Math., 55, Dekker, New York, 1980.

11. J. Herzog and T. Hibi, "Discrete polymatroids," J. Algebraic Combin. 16(3) (2002), 239-268.

12. J. Herzog and T. Hibi, G. Restuccia "Strongly Koszul algebras," Math. Scand. 86(2) (2000), 161-178.

13. J. Herzog and N.V. Trung, "Gröbner bases and multiplicity of determinantal and Pfaffian ideals," Adv. Math. 96(1) (1992), 1-37.

14. T. Hibi, "Distributive lattices, affine semigroup rings and algebras with straightening laws," Commutative Algebra and Combinatorics (Kyoto, 1985), pp. 93-109, Adv. Stud. Pure Math., 11, North-Holland, Amsterdam, 1987. 
15. T. Hibi and K. Watanabe, "Study of three-dimensional algebras with straightening laws which are Gorenstein domains. I," Hiroshima Math. J. 15(1) (1985), 27-54.

16. T. Hibi and K. Watanabe, "Study of three-dimensional algebras with straightening laws which are Gorenstein domains. II," Hiroshima Math. J. 15(2) (1985), 321-340.

17. T. Hibi, "Study of three-dimensional algebras with straightening laws which are Gorenstein domains. III," Hiroshima Math. J. 18(2) (1988), 299-308.

18. J. Oxley, Matroid Theory, Oxford University Press, Oxford, 1992.

19. J. Roos, "A description of the homological behaviour of families of quadratic forms in four variables," in Syzygies and Geometry, Boston 1995, A. Iarrobino, A. Martsinkovsky, and J. Weyman (Eds.), Northeastern Univ., 1995, pp. 86-95.

20. B. Sturmfels, Gröbner Bases and Convex Polytopes, Amer. Math. Soc., Providence, RI, 1995.

21. R. Villarreal, "Monomial algebras," Monographs and Textbooks in Pure and Applied Mathematics, Vol. 238. Marcel Dekker, Inc., New York, 2001.

22. K. Watanabe, "Study of algebras with straightening laws of dimension 2," Algebraic and Topological Theories (Kinosaki, 1984), Kinokuniya, Tokyo, 1986, pp. 622-639.

23. K. Watanabe, "Study of four-dimensional Gorenstein ASL domains. I. Integral posets arising from triangulations of a 2-sphere," Commutative Algebra and Combinatorics (Kyoto, 1985), Adv. Stud. Pure Math., Vol. 11, North-Holland, Amsterdam, 1987, pp. 313-335.

24. V. Weispfenning, “Comprehensive Gröbner bases,” J. Symbolic Comput. 14(1) (1992), 1-29.

25. D. Welsh, Matroid Theory, Academic Press, London, 1976.

26. N. White, "A unique exchange property for bases," Linear Algebra Appl. 31 (1980), 81-91. 